\title{
Percepción de los Estudiantes de Ciencias Químicas sobre sus Equipos de Trabajo
}

\author{
Jorge A. Ramírez, María G. Alejo, Rebeca Jiménez, Salvador Marmolejo \\ Facultad de Ciencias Químicas, Universidad Autónoma de San Luis Potosí \\ Manuel Nava \# 6, Zona Universitaria, 78210; San Luis Potosí, S. L. P.-México \\ (e-mail: jrt@uaslp.mx; _galejog@uaslp.mx; rjimenezh@fcq.uaslp.mx; smarmolejo@uaslp.mx)
}

Recibido Jul. 04, 2012; Aceptado Ago. 07, 2012; Versión final recibida Feb. 11, 2013

\begin{abstract}
Resumen
Se presenta un estudio sobre la percepción de los estudiantes del funcionamiento de sus equipos de trabajo en materias semestrales en la Facultad de Ciencias Químicas de la Universidad Autónoma de San Luis Potosí en México. Se aplicó una encuesta a 150 estudiantes de los niveles inicial, medio y terminal de las carreras de Ingeniería Química e Ingeniería en Alimentos. Los criterios evaluados fueron integración, organización, interacción, funcionamiento y calidad. De acuerdo a los resultados se observó que a medida que avanzan en su nivel académico disminuye la calificación de los criterios evaluados. Estos resultados pueden ser debidos a diversos factores y abre el espacio para nuevas investigaciones que den como resultado acciones concretas para el mejoramiento de las técnicas didácticas y para sensibilizar a estudiantes y profesores sobre la aplicación eficiente de las mejoras.
\end{abstract}

Palabras clave: percepción de grupo, equipos de trabajo, técnicas didácticas, enseñanza universitaria

\section{Chemical Science Students Perception of Their Team Work}

\begin{abstract}
This paper presents a study on the perception that students have on the operation of their team work in term courses of the Faculty of Chemical Sciences of the Universidad Autónoma de San Luis Potosí in México. A survey was applied to 150 students of initial, middle and terminal levels of Chemical Engineering and Food Engineering. The criteria that were evaluated were: integration, organization, interaction, performance and quality. The results indicate that as the students advance in their academic level qualification of all these aspects decreases. These results may be due to various factors, and opens up the space for new research that will result in concrete actions to improve teaching technics and to sensibilize students and teachers on the efficient application of those improving measures.
\end{abstract}

Keywords: perception of group, work team, teaching technics, competence, university teaching. 


\section{INTRODUCCIÓN}

La transformación y actualización tecnológica de los campos profesionales genera nuevas estrategias en la educación actual, por lo tanto, la versatilidad es cada vez más una característica fundamental en la formación profesional. Aceptar la naturaleza compleja de las competencias profesionales implica concebir al egresado como persona que se expresa y se desarrolla en el ejercicio de la profesión. La formación en competencias profesionales es uno de los objetivos esenciales de la universidad actual; sin embargo, es interesante destacar que el interés por ellas no surge en el contexto educativo, sino en el mundo del trabajo desde la década de los 70's y es, a partir de los estudios de diferentes autores, que comienza una búsqueda orientada al hallazgo de criterios científicos explicativos de la eficiencia de las personas en el desempeño laboral (González y González, 2008).

Las condiciones actuales en las que ha de desempeñarse el profesional, la globalización y la sociedad del conocimiento, plantean exigencias a la formación de profesionales universitarios, que permitan garantizar no sólo el dominio de los conocimientos para el desempeño específico de una profesión en un contexto histórico-social determinado, sino también y, fundamentalmente, la formación en valores, habilidades y recursos personales que motiven al egresado a desempeñarse con eficiencia, autonomía, ética y compromiso social en los contextos, heterogéneos y cambiantes, en donde va a desarrollarse (Delors, 1996). Todo ello exige a la universidad centrar la atención a la formación en competencias genéricas y profesionales.

El enfoque basado en competencias puede ser el puente entre el paradigma de la educación tradicional y la revolución del aprendizaje, esto causaría que muchas instituciones examinen lo que actualmente ofrecen, ya que en la actualidad existe una brecha considerable entre las intenciones y las acciones (Voorhees, 2001).

La reforma curricular universitaria que tiene lugar en la actualidad en el proceso de convergencia europeo de educación superior, está dirigido a lograr competitividad, empleabilidad y movilidad para los profesionales en Europa a partir de titulaciones fácilmente comparables y comprensibles centrando la atención en la formación en competencias profesionales (Beneitone et al. 2007). En la concepción de las competencias genéricas (comunes a todas las profesiones) en el proyecto Tuning (2007), se reconoce a la capacidad para el trabajo en equipo, la habilidad para el manejo de las relaciones interpersonales y el compromiso ético, entre otras. Existen competencias que también constituyen la base del aprendizaje y representan la característica innata de las experiencias de cada individuo sobre las que se pueden cimentar las diferencias en rasgos y características, lo que explica el por qué la gente aprende a través diferentes experiencias y adquieren diferentes niveles y tipos de destrezas, habilidades y conocimientos (Voorhees, 2001).

Dentro de las competencias genéricas, el trabajo en equipo es fundamental, dado que el ser humano convive todos los días con personas diferentes, circunstancia que lo conduce a desarrollar habilidades que le permiten realizar trabajos interactuando con otros individuos. La acción grupal suele ser más segura y efectiva que la gestión individual o la simple adición de acciones individuales. La colaboración mediante el trabajo en equipo, permite analizar problemas que son comunes, con mayor y mejor criterio, por lo que es importante cuestionarse, ¿cómo podemos desarrollar la confianza de nuestros estudiantes en sus habilidades de trabajo en equipo? (Felder et al. 2000).

Desarrollar la competencia de trabajo en equipo, como toda competencia, requiere de una transmisión y es importante poder integrar el conjunto de conocimientos que se han adquirido a lo largo del tiempo para ponerlos en práctica (Echeverría, 2002). No es sólo saber que el trabajo en equipo es cooperar con los compañeros, sino que se tiene que saber hacer, saber estar y saber ser (Echeverría et al. 2002; Irigoyen et al. 2011). Es un grado más en esta estructura compleja que culmina con la puesta en práctica de todo aquello que se sabe, o del conjunto de recursos adquiridos (Torrelles et al. 2011).

Las habilidades relacionadas con el trabajo en equipo, comprende los puntos de vista de los demás, dando paso a la delegación y aceptación de responsabilidades, haciendo frente a conflictos interpersonales que surgen inevitablemente, lo cual puede ser vital para el éxito de un proyecto en equipo. Ser consciente de las necesidades de los demás y considerarlos al tomar decisiones puede ser la esencia del trabajo en equipo, lo cual es sin duda un requisito previo para el funcionamiento profesional y ético (Rugarcía et al. 2000)

Un equipo de trabajo consiste en una agrupación de personas trabajando juntas que comparten percepciones, tienen una propuesta en común, están de acuerdo con los procedimientos de trabajo, cooperan entre sí, aceptan un compromiso y resuelven sus desacuerdos en discusiones abiertas, para lograr un objetivo en común (Clausse, 2012). Lo anterior, no surge automáticamente, sino que debe irse 
construyendo poco a poco. Se habla entonces de una acción colaborativa donde la discusión no es el objetivo, sino el medio (Barrios et al. 2004).

En este sentido, el Equipo de evaluación de Escuelas de Negocios y Tecnologías (2009), define las habilidades elementales para realizar un buen trabajo en equipo, que son:

Integración. Un trabajo de equipo exitoso comienza con la selección de cada uno de los integrantes, para convertirla luego en un equipo de trabajo. Por "formación de un equipo de trabajo" se entienden aquellas actividades tendientes a convertir a un grupo en una unidad de trabajo capaz de funcionar a su nivel óptimo. Una formación adecuada del equipo ayuda a establecer una organización y cultura de trabajo apropiadas y acelera la acumulación de experiencia. Los equipos que fueron formados a través de un proceso apropiado superan en desempeño a los equipos que se forman simplemente poniendo al azar a varias personas a trabajar en una misma tarea.

Organización. Un equipo de trabajo debe organizarse en forma simple mediante el arreglo y utilización de los recursos materiales y humanos para el logro de metas. Las actividades de la organización deberán ser de información, diagnóstico y planeación de la acción. Un proceso de desarrollo posterior será más efectivo si en la organización se toma en cuenta que los miembros tengan claras las razones por las que participan en una determinada actividad y que la asistencia de los miembros forme una unidad de trabajo completa logrando coordinación, planeación, metas compartidas y toma de decisiones en común.

Interacción. Para que la interacción entre los que forman un equipo de trabajo sea efectiva, es necesaria la capacidad de escucha entre los miembros, crear un ambiente de confianza y apertura, eliminar temores, valorar su diversidad, dar el ejemplo, comunicar el objetivo y misión al equipo, delegar, instruir, aconsejar, guiar, estimular creatividad, toma de riesgos y mejoramiento continuo de todo por todos, compartir información, motivar, crear las condiciones para que las personas sean más capaces de hacer su trabajo y obtener resultados de calidad.

Funcionamiento. El buen funcionamiento de un equipo de trabajo está íntimamente relacionado con la interacción que hay entre sus miembros y para que sea óptimo, además de los puntos mencionados en Interacción, deberá considerarse contar con un objetivo/misión compartido, que todos conocen y, por lo mismo, están comprometidos a cumplir ya que los integrantes participaron en la definición de las metas.

Calidad de los resultados. Es evaluada a través del cumplimiento de objetivos y metas, manejo de tiempo, si el resultado es integrado, coherente y de alta calidad. Además, un trabajo en equipo de calidad, permite la retroalimentación como una herramienta colaborativa que fomenta el aprendizaje autónomo, lo cual conduce a una educación de formación para la vida y no sólo para el momento en que se desarrolla la actividad.

Dada la importancia de la educación basada en competencias, la Universidad Autónoma de San Luis Potosí ha iniciado un programa con este enfoque que permita a sus futuros egresados incorporarse con éxito en el ámbito laboral. Acorde a lo anterior, la Facultad de Ciencias Químicas a partir de los Foros de Empleadores (2005) y Egresados (2011), así como en un estudio previo realizado por Marmolejo et al. (2010), donde identificaron la problemática existente en relación a la capacidad para trabajar en equipo. Debido a lo anterior se planteó la necesidad de analizar la percepción que los estudiantes tienen sobre el trabajo en equipo. Es de suponer que los equipos de trabajo de los estudiantes de las carreras de ingeniería química e ingeniería en alimentos que cursan las materias de nivel terminal mejoran su integración, organización, interacción, funcionamiento y calidad, en comparación con los equipos de trabajo de los alumnos que cursan nivel inicial y medio.

\section{METODOLOGÍA}

El presente estudio se realizó en la Facultad de Ciencias Químicas de la Universidad Autónoma de San Luis Potosí (FCQ/UASLP), la muestra seleccionada consideró 150 estudiantes de las carreras de Ingeniería Química e Ingeniería en Alimentos que se ofrecen en la Facultad. Del total de la muestra, 50 alumnos pertenecían a los dos primeros semestres del laboratorio de Química General I y II (iniciales), 50 a los laboratorios de Fisicoquímica y Separaciones mecánicas (Medio) y 50 alumnos del nivel terminal, de las materias de Control de Procesos y Desarrollo de Nuevos Productos. La muestra representa al menos el 85\% de los estudiantes de los programas de Ingeniería Química e Ingeniería en Alimentos en cada uno de los diferentes niveles.

Con la finalidad de obtener datos sobre la percepción que los estudiantes tienen sobre el equipo de trabajo, se elaboró una rúbrica que permitiera recuperar la información con criterios precisos sobre las habilidades elementales para realizar un buen trabajo en equipo. Se hizo una revisión de diferentes rúbricas para 
evaluar el trabajo en equipo y se eligió el modelo de rúbrica del Equipo de Evaluación del Colegio de Negocios y Tecnologías (College of Business and Technology's Assesment Team), de la Universidad de Minnesota, modificada y adecuada, a fin de evaluar los siguientes criterios del trabajo en equipo: (1) Integración, (2) Organización, (3) Interacción, (4) Funcionamiento y (5) Calidad de resultados. La rúbrica aplicada se muestra en la figura 1.

Las variables que mide la rúbrica elegida cumplen con las intenciones de la investigación. Las adaptaciones de la rúbrica empleada para la evaluación, se realizaron de la original considerando el enfoque personal sobre trabajo en equipo de cada aseveración hacia la percepción del equipo de trabajo. A fin de verificar la claridad de la rúbrica, se aplicó una prueba piloto a 10 estudiantes.

Posteriormente, la rúbrica fue aplicada, por los profesores de las asignaturas, de los diferentes grupos al finalizar el semestre agosto- diciembre del 2011, solicitando a los estudiantes que expresaran la percepción que tuvieron, en cuanto al trabajo desarrollado en sus equipos, en las diferentes materias y laboratorios.

El cuestionario constó de 27 aseveraciones, de las cuales, dos correspondieron al criterio Integración del equipo; cinco a Organización; nueve a Interacción; siete a Funcionamiento y, finalmente, cuatro a Calidad de los resultados. En cada pregunta se solicitó que seleccionaran una de las siguientes opciones de respuesta: No aplica, No satisface expectativas, Cumple con expectativas y Supera expectativas, a las cuales con el fin analizar gráficamente, se asignaron los valores 0, 1, 2 y 3, respectivamente (Figura 1).

\section{TRABAJO EN EQUIPO}

Materia

Semestre

La presente encuesta tiene como objetivo retroalimentar el proceso de trabajo en equipo, por lo que se te pide contestes de manera HONESTA EL DESEMPEÑO DE TU EQUIPO DE TRABAJO.

\section{El equipo de trabajo}

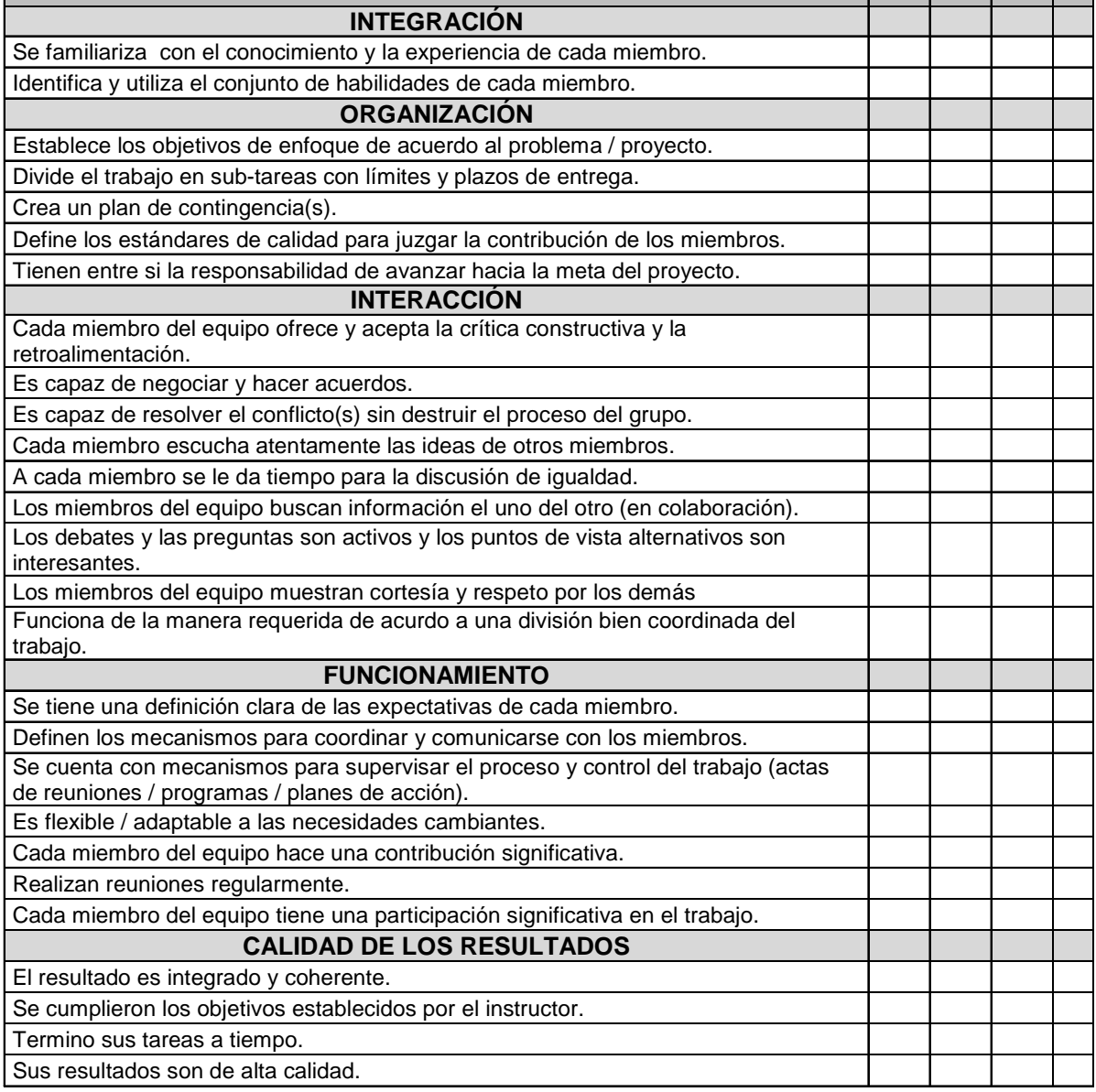

Fig. 1. Rubrica empleada en el estudio. 


\section{RESULTADOS Y DISCUSIÓN}

Para analizar los datos, se asignó el valor de cero, a la opción de respuesta "No aplica"; 1, a "No satisface"; 2, a "Cumple" y 3, a "Supera". A fin de reducir el efecto de tener diferente número de preguntas, para evaluar los criterios, se calculó la media (suma de calificaciones, dividida entre el número de preguntas de cada criterio). De acuerdo a lo anterior puede observarse que en el nivel Inicial las medias de calificaciones de los criterios de percepción del trabajo en equipo, oscilaron entre, 2.25 y 2.31, mientras que en el Medio 1.95 a 2.20 y entre 1.95 y 2.12, en el nivel Terminal (Figura 2). Cabe destacar que aunque no exista una diferencia importante entre los diferentes promedios por criterio, puede observarse una tendencia a disminuir, de acuerdo al nivel académico, es decir, en el nivel inicial para los cinco criterios, se obtuvieron los promedios más altos y los más bajos correspondieron al nivel terminal. Analizando de forma general, por criterio, la calificación de integración disminuyó de 2.31, en el nivel Inicial, a 2.03 en el Terminal. El criterio Organización, disminuyó de 2.31, en el nivel inicial, a 1.95 en el nivel Terminal (aunque se obtuvo el mismo valor en el nivel Medio). La calificación media para Interacción del equipo, se redujo, de 2.29 a 2.11, en el Terminal. En funcionamiento y calidad, también reducen, de 2.25 y 2.27, a 2.05 y 2.12, respectivamente.

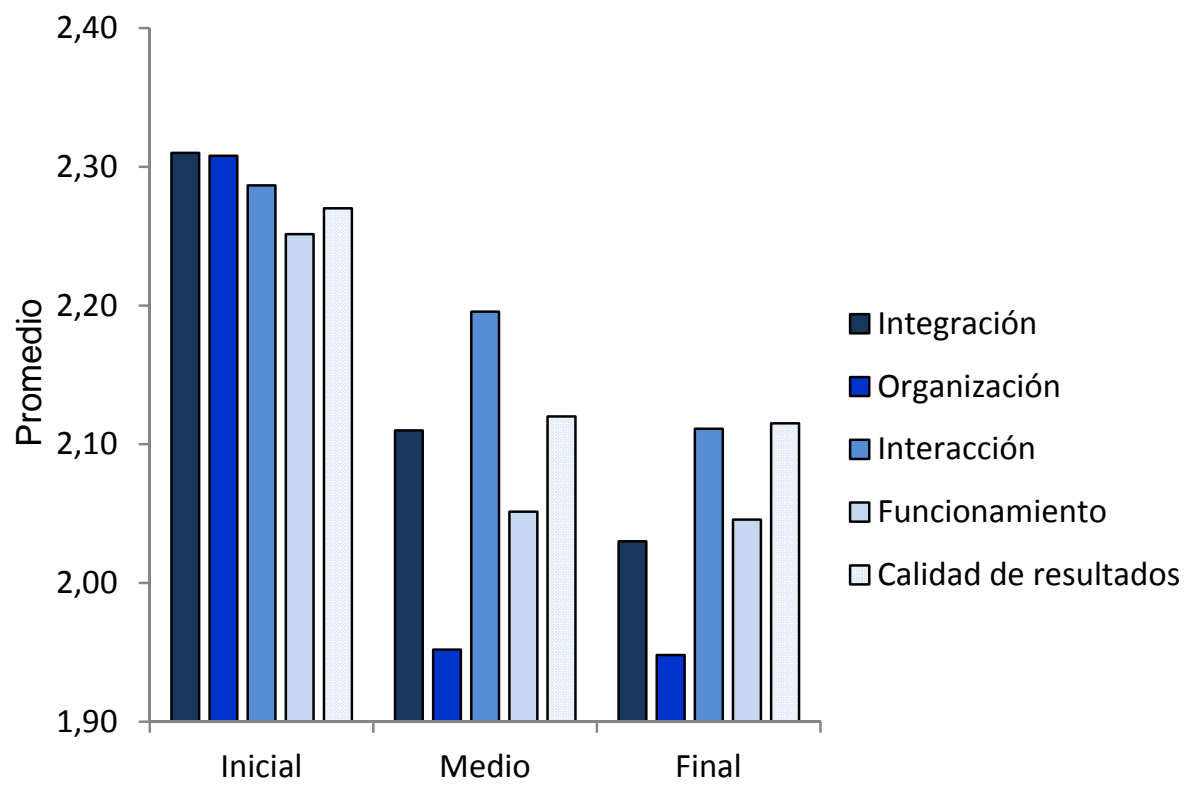

Nivel Académico

Fig. 2. Criterios y promedio de calificaciones en los 3 niveles analizados.

En los tres niveles estudiados, puede observarse la disminución en las medias de calificaciones de las variables estudiadas (Integración, Organización, Interacción, Funcionamiento y Calidad), Específicamente, las diferencias obtenidas entre las medias de calificaciones de los criterios, Organización (de 2.31 a 2.03), Interacción (de 2.29 a 2.05) y Calidad (2.27 a 2.12), en los niveles Inicial y Terminal. Esta disminución en las calificaciones, probablemente pueda deberse a que en el nivel inicial los estudiantes ingresan a la licenciatura con las competencias adquiridas en grados escolares anteriores, ya que las reformas educativas en la educación media superior, iniciaron en el ciclo escolar 2008-2009 (SEP, 2008), por lo que los estudiantes del nivel Inicial son de los primeros egresados con el marco curricular basado en el desarrollo de competencias. En la figura 3, se representan, de forma general, las opiniones sobre las opciones de respuesta de los cinco criterios, en los niveles encuestados (Inicial, Medio y Terminal). Se obtuvieron un total de 4050 respuestas, de las cuales el mayor porcentaje de calificación correspondió a la opción de "Cumple" (61.6\%). Sin embargo, las opciones "Supera" y "No satisface", también fueron consideradas de manera importante por los estudiantes encuestados (27.1 y $10.1 \%$, respectivamente).

En la figura 4 se representa el análisis de calificaciones, por nivel académico, mostrando que el mayor porcentaje de respuestas, consideró que "Cumplen" con las distintas actividades relacionadas con el trabajo en equipo, al obtener los resultados mayores de los tres Niveles estudiados (68.8, 54.8 y $61.2 \%$, para los niveles Inicial, Medio y terminal, respectivamente). Sin embargo, en el criterio "No satisface" el cumplimiento, aumentó considerablemente del nivel Inicial al Medio, de 1.5 a 14.6\%. La opción "Supera" disminuyó de 29.6 a $23.0 \%$ del nivel Inicial al terminal, respectivamente. 


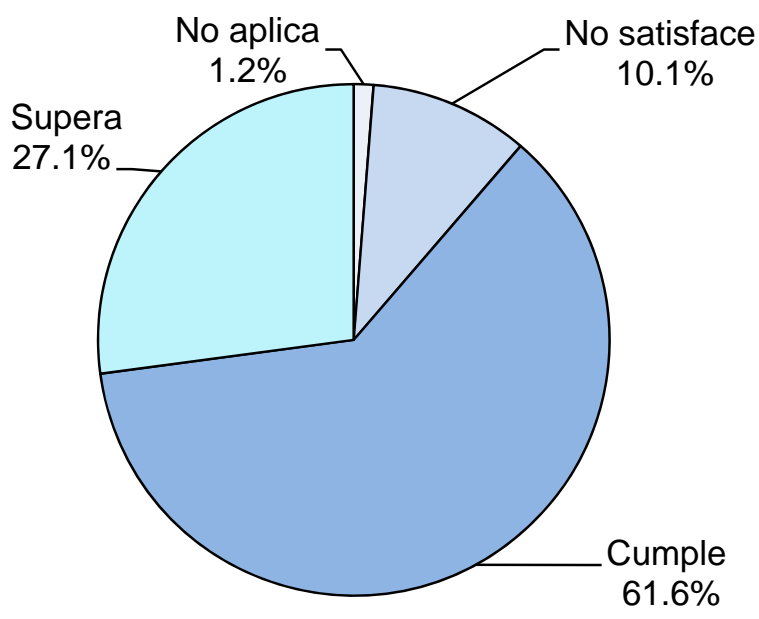

Fig. 3. Porcentaje de respuestas de la evaluación general de trabajo en equipo.

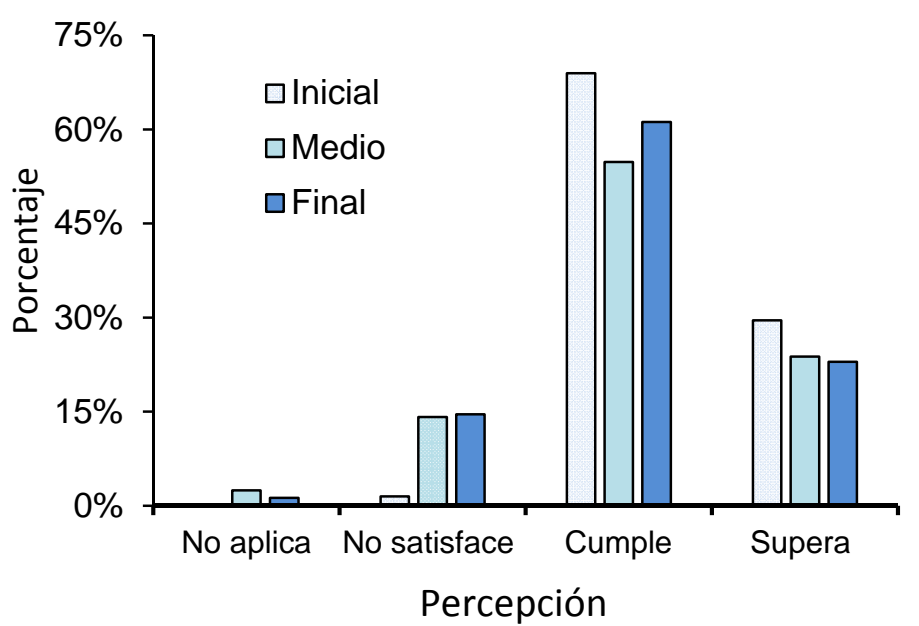

Fig. 4. Distribución de respuestas por nivel.

Aunque los resultados obtenidos, no apoyan la hipótesis planteada, probablemente sí estén de acuerdo con la opinión de Felder et al. (2000), quienes consideran que muchos profesores creen que mediante el desarrollo de un experimento de laboratorio o un diseño de proceso, realizado por tres o cuatro estudiantes, de alguna manera el proyecto debería permitirles desarrollar las habilidades de liderazgo, control del tiempo, comunicación y resolución de conflictos, habilidades que caracterizan a los equipos de alto rendimiento. Desafortunadamente, no es así, lo que suele ocurrir, es que uno o dos estudiantes realizan la mayor parte o la totalidad del trabajo, siendo que todos cursan el mismo grado. Esta situación no promueve el desarrollo de habilidades de trabajo en equipo y manifiesta la importancia de la labor del profesor para el buen funcionamiento del equipo.

Existen otros factores que podrían considerarse, entre los que destacan: metodología empleada, número de integrantes, género y su capacidad para el manejo de conflictos (Gencosman y Dogru, 2012), entre otros. Sin embargo, aunque los resultados de este estudio, indican una disminución de las habilidades y actitudes del trabajo en equipo, se observa una transición durante su formación profesional, lo cual probablemente pueda deberse a cambios en su criterio y/o al desarrollo de otras habilidades y actitudes adquiridas durante sus estudios universitarios. La capacidad para trabajar en equipo es una habilidad muy apreciada en el ámbito laboral y que los profesores pueden cultivar en los estudiantes, a través de la aplicación de técnicas de aprendizaje colaborativo (Regalado-Méndez et al. 2011; Lorenzo, Fernández y Carro, 2011; Domínguez y Michel, 2010; González y Génova, 2008; Felder y Brent, 2004, 1994; Bedard, 2001, Woods et al. 2000). Además, se ha observado que con el desarrollo de esta habilidad, se incrementan los logros académicos, así como una mejor actitud para el aprendizaje (Espey, 2010) y consecuentemente mejoran su autoestima. El aprendizaje en equipo ayuda a desarrollar la creatividad, la flexibilidad y el diálogo al interior de los equipos, resulta fundamental para determinar la capacidad de aprendizaje de la organización (Pérez; Bustamante y Maldonado, 2009). Por estas razones, potenciar la capacidad de autoaprendizaje y aplicarla en el trabajo colaborativo debe ser objetivo primordial en los programas de estudio. 
A fin de lograr la comprensión integral y la importancia del trabajo en equipo, se requiere del compromiso y colaboración de los profesores de todos los cursos, así como concientizar a los estudiantes de la necesidad de involucrarse en su proceso de mejora personal y profesional mediante la participación (Ugarte y Naval, 2010) y así promover la mejora de estas habilidades y actitudes, las cuales llevarán al desarrollo de esta competencia, esperando el impacto deseado en los egresados. No obstante, para lograr esto, es requisito que se tenga la concepción en relación al enfoque por competencias y no hacerlo simplemente a través de interacciones didácticas, ya que debe haber una implementación pertinente con fundamentos epistemológicos (Ruiz, 2009). Cabe destacar que en diversos estudios realizados en diferentes áreas y niveles, se ha demostrado que el aprendizaje cooperativo tiene un efecto positivo en varios procesos mentales, como son: enseñanza-aprendizaje, emocionales y cognitivos, memorización, aplicación de estrategias de alto nivel cognitivo, participación en clase y autoestima (Lee, Lim y Ng, 1997; Keyser, 2000; Lee, $\mathrm{Ng}$ y Phang, 2002; Bilgin, 2006). El estudio efectuado en los estudiantes de la Facultad de Ciencias Químicas/Universidad Autónoma San Luis Potosí, es fundamental, ya que ayudó a identificar la realidad de los estudiantes respecto a su percepción sobre el trabajo colaborativo, lo cual da la pauta para sensibilizar profesores, diseñar e implementar estrategias en los diferentes cursos durante su formación profesional.

\section{CONCLUSIONES}

Se identificó la realidad de los estudiantes de diferentes niveles, en cuanto a su percepción del trabajo en equipo, destacando que los de primer semestre, mostraron mejor percepción del trabajo en equipo, que los de semestres terminales, encuestados en el mismo ciclo escolar. Esta disminución en la evaluación de la capacidad para el trabajo colaborativo y habilidades relacionadas durante su formación profesional, son un indicativo importante que deberá considerarse como área de oportunidad de mejora, en los programas de estudio. También será importante promover la capacitación de profesores para diseñar e implementar estrategias de aprendizaje colaborativo en los diferentes cursos, enfatizando la necesidad y concientizándolos sobre la importancia del fomentar el trabajo en equipo. De acuerdo a los resultados, es recomendable continuar con este tipo de estudios, aplicando la metodología más adecuada para evaluar, de manera más objetiva, el desarrollo de competencias profesionales, desde una perspectiva individual.

\section{REFERENCIAS}

Barrios, N.; Castillo, M.C.; Fajardo, F.; Rojas, J.; Nova, A.J. El aula, un escenario para trabajar en equipo. Tesis de Maestría en Educación, Pontificia Universidad Javeriana, Bogotá, Colombia (2004).

Bedard, A., Creating and Implementing Competency-Based Learning Models. New Directions for Institutional Research, No. 110, Summer. 83-95 (2001).

Beneitone, P.; Esquetini, C.; González, J.; Maletá, M.; Siufi, G.; Wagenaar, R., Reflexiones y perspectivas de la educación superior en América Latina. Informe Final Proyecto Tuning América Latina 2004-2007. Publicaciones Universidad de Deusto, Bilbao, España (2007).

Bilgin, I., The Effects of Hands-on Activities Incorporating a Cooperative Learning Approach on Eight Grade Students' Science Process Skills and Attitudes toward Science, Journal of Baltic Science Education, 1(9), 27-37 (2006).

Clausse, A., Organización de Equipos de Trabajo de Investigación y Desarrollo, ISISTAN, Facultad de Ciencias Químicas, Universidad Nacional del Centro, Argentina. (En línea)

http://www.exa.unicen.edu.ar/catedras/manageit/docs/Equipos.pdf. Acceso: 10 Febrero (2013).

Delors, J., Informe a la UNESCO de la Comisión Internacional sobre la Educación para el Siglo XXI. Madrid, España. Santillana, UNESCO (1996).

Domínguez, O. J. y Michel, R. L., Mejoras en el Desarrollo del Proyecto Final para Estudiantes de Ingeniería Química. Formación Universitaria Vol. 3(4), 47-52 (2010).

Echeverría, B., Gestión de la competencia de Acción Profesional, Revista de Investigación Educativa, Vol. 20, No. ,1 7-43, Universidad de Barcelona (2002).

Espey, M., Valuing Teams: What Influences Student Attitudes? Journal North American College \& Teachers of Agriculture. March. 31-40 (2010).

Felder, R.; Woods, D.; Stice J.; Rugarcía, A., The Future of Engineering Education II. Teaching methods that work, Chem. Eng. Education, 34 (1), 26-39 (2000). 
Felder, R. M.; Brent, R., The ABC's of Engineering Education: ABET, Bloom's taxonomy, Cooperative learning, and so on. Proceedings of the 2004 American Society for Engineering Education Annual Conference \& Exposition, American Society for Engineering Education. Session 1375 (2004).

Felder, R. M.; Brent, R., Cooperative learning in technical courses: procedures, pitfalls, and payfoffs. National Science Foundation Division of Undergraduate Education Grant, October 1994. 1-21 (1994). http://www4.ncsu.edu/unity/lockers/users/f/felder/public/Papers/Coopreport.html Acceso: 10 Febrero (2013).

Gencosman, T.; Dogru, M., Effect of student teams-achievement divisions technique used in science and technology education on self-efficacy, test anxiety and academic achievement. Journal of Baltic Science Education, Vol. 11, No. 1. 43 - 54 (2012).

González, M. R.; Génova, G., Innovación docente a la luz de Bolonia: Trabajo en equipo y revisiones cruzadas para convertir al alumno en protagonista de su proceso de aprendizaje. Teoría de la Educación. Educación y Cultura en la Sociedad de la Información, Vol. 9, No. 1, 126-141, Febrero (2008). http://redalyc.uaemex.mx/src/inicio/ArtPdfRed.jsp?iCve=201017338008 Acceso: 10 Febrero (2013).

González, V.; González, R., Competencias Genéricas y Formación Profesional: Un análisis desde la docencia universitaria. 2008. (En línea) http://www.rieoei.org/rie47a09.htm. Acceso: 10 Febrero (2013).

Irigoyen, J. J.; Jiménez, M. Y.; Acuña, K. F., Competencias y Educación Superior. Revista Mexicana de Investigación Educativa. Vol. 16, No. 48, 243-266. Enero-Marzo (2011).

Keyser, M. W., Active Learning and Cooperative Learning: Understanding the Difference and Using Both Styles Effectively. Research Strategies, 77(1), 35-44 (2000).

Lee, C.; Lim, T. y Ng, M., Affective Outcomes of Cooperative Learning in Social Studies. Asia Pacific Journal of Education, 77(1), 57-75 (1997).

Lee, C.; Ng, M. y Phang R., Effects of Cooperative Learning on Elementary School Children in Singapore. Asia Pacific Journal of Education, 22 (1), 3-15 (2002).

Lorenzo, R. A.; Fernández, P. y Carro A M., Experiencia en la Aplicación del Aprendizaje Basado en Problemas en la Asignatura Proyecto de Licenciatura en Química. Formación Universitaria. Vol. 4(2), 37-44 (2011).

Marmolejo, S.; Jiménez, R.; Alejo, M.G.; Ramírez, J.A., Calidad del trabajo en equipo. Memorias del XXXI Encuentro Nacional de la AMIDIQ. México (2010).

Pérez, I.; Bustamante, S.; Maldonado, M., El trabajo en equipo y el desarrollo de habilidades conversacionales en organizaciones educativas. Omnia, Vol. 15, No. 3, 78-96 (2009). http://revistas.luz.edu.ve/index.php/omnia/article/viewFile/8726/8699 . Acceso: 10 Febrero (2013).

Regalado-Méndez, A.; Peralta-Reyes, E. y Báez-González, J. G., Aprendizaje Basado en Competencias Aplicado a una Asignatura de Transferencia de Calor. Formación Universitaria. Vol. 4(1), 13-18 (2011).

Rugarcía, A.; Felder, R.; Woods, D.; Stice, J., The future of engineering education I. A vision for a new century, Chem. Eng. Education, 34 (1), 16-25 (2000).

SEP (Secretaría de Educación Pública). Reforma Integral de la Educación Media Superior, Diario Oficial de la Federación. 1ª. Sección. México. 21 Octubre (2008).

Torrelles, C.; Coiduras, J.; Isus, S.; Carrera, F.; Paris, G.; Cela, J., Competencia de Trabajo en Equipo: Definición y categorización. Revista de currículum y formación de profesorado, vol. 15, No. 3, 329-344 (2011).

Ugarte, C., Naval, C. Desarrollo de competencias profesionales en la educación superior. Un caso docente concreto, Revista Electrónica de Investigación Educativa, Número especial 2 (2010) http://redie.uabc.mx/NumEsp2/contenido-ugarte.html_Acceso: 10 Febrero (2013).

University of Minnesota Duluth (UMD). College of Business and Technology's assesment team. Directions for Instructor use of the teamwork assessment rubric. (En línea).

http://www.d.umn.edu/vcaa/assessment/documents/Teamworkrubric.pdf. Acceso: 10 Febrero (2013). 
Voorhees, R. A., Competency-Based Learning Models: A Necessary Future New Directions for Institutional Research, No. 110, Summer. 5-13 (2001).

Woods, D.; Felder, R.; Rugarcía, A.; Stice, J., The Future of Engineering Education III. Developing critical skills, Chem. Eng. Education, 34 (2), 108 - 117 (2000). 
